Subject Index to Volume $22-1995$

Aging

Environmental Exposures in Elderly Canadians with Parkinson's Disease 232 (AUG)

"Unexplained" Delayed Death From Fungal Meningitis After Meningioma Resection 239 (AUG)

Selective Sparing of Human Nucleus Accumbens in Aging and Anoxia 290 (NOV)

AIDS

Sensorimotor Neuropathy and Abnormal Vitamin B $_{12}$ Metabolism in Early HIV Infection 43 (FEB)

Bilateral Central Ptosis in Acquired Immunodeficiency Syndrome 52 (FEB)

HIV-1 Associated Dementia: Clinical Features and Pathogenesis 92 (MAY)

Alzheimer Disease

Canadian Guidelines for the Development of Antidementia Therapies: a Conceptual Summary 62 (FEB)

Amyloid

Dépôts Amyloïdes Intraligamentaires dans la Sténose du Canal Rachidien 164 (MAY)

Aneurysms

Surgery of Unruptured, Asymptomatic Aneurysms: A Decision Analysis 30 (FEB)

Risk of Intracranial Aneurysms in Families with Subarachnoid Hemorrhage 121 (MAY)

Protection of the Brain after Aneurysmal Rupture 177 (AUG)

Ruptured and Unruptured Intracranial Aneurysms-Surgical Outcome 187 (AUG)

Letter to the Editor: Risk of Intracranial Aneurysms in Families with Subarachnoid Hemorrhage 333 (NOV)

Reply 333 (NOV)

Anoxia

Selective Sparing of Human Nucleus Accumbens in Aging and Anoxia 290 (NOV)

Astrocytomas

Loss of Heterozygosity Analysis of Chromosomes 9, 10 and 17 in Gliomas in Families 17 (FEB)

Autonomic Nervous System

Long Term Treatment of Intractable Reflex Sympathetic Dystrophy with Intrathecal Morphine 153 (MAY)

Paradoxical Autonomic Response to Procyclidine in the Neuroleptic Malignant Syndrome 244 (AUG)

Baclofen

Intrathecal Baclofen Therapy for Adults with Spinal Spasticity: Therapeutic Efficacy and Effect on Hospital Admissions 22 (FEB)

\section{Blood Flow}

Experimental Delayed Postischemic Spinal Cord Hypoperfusion After Aortic Crossclamping 202 (AUG)
Can J Neurol Sci

Letter to the Editor: What's in a Name? 247 (AUG)

Reply from the Editor 247 (AUG)

Cerebellar Dysfunction

Myoclonic-like Finger Microdisplacements in Patients with Cerebellar Deficits 144 (MAY)

\section{Cerebellum}

Brain Herniation: A Revision of Classical Concepts 83 (MAY)

Myoclonic-like Finger Microdisplacements in Patients with Cerebellar Deficits 144 (MAY)

\section{Cerebral Blood Flow}

Protection of the Brain after Aneurysmal Rupture 177 (AUG)

\section{Cerebral Edema}

Brain Herniation: A Revision of Classical Concepts 83 (MAY)

\section{Cerebrovascular Disease}

Editorial 3 (FEB)

Surgery of Unruptured, Asymptomatic Aneurysms: A Decision Analysis 30 (FEB)

Canadian Guidelines for the Development of Antidementia Therapies: a Conceptual Summary 62 (FEB)

Risk of Intracranial Aneurysms in Families with Subarachnoid Hemorrhage 121 (MAY)

Protection of the Brain after Aneurysmal Rupture 177 (AUG)

Ruptured and Unruptured Intracranial Aneurysms-Surgical Outcome 187 (AUG)

Mechanisms Underlying Functional Recovery Following Stroke 257 (NOV)

\section{Chemotherapy}

$\mathrm{O}^{6}$-Methylguanine-DNA Methyltransferase in Tumors and Cells of the Oligodendrocyte Lineage 111 (MAY)

Chorea

Huntington's Disease: Recent Advances in Diagnosis and Management 5 (FEB)

Coma

Bilateral Temporal Lobe MRI Changes in Uncomplicated Hypoglycemic Coma 56 (FEB)

Brain Herniation: A Revision of Classical Concepts 83 (MAY)

$\beta$-Endorphin in Multiple Trauma Victims 160 (MAY)

\section{Coma Scale}

Myoclonic-like Finger Microdisplacements in Patients with Cerebellar Deficits 144 (MAY)

Dementia

Editorial 3 (FEB)

Canadian Guidelines for the Development of Antidementia Therapies: a Conceptual Summary 62 (FEB)

HIV-1 Associated Dementia: Clinical Features and Pathogenesis 92 (MAY)
Depression

Screening for Major Depression in the Early Stages of Multiple Sclerosis 228 (AUG)

\section{Diabetes Mellitus}

Bilateral Temporal Lobe MRI Changes in Uncomplicated Hypoglycemic Coma 56 (FEB)

Nerve Microvessel Changes in Diabetes are Prevented by Aldose Reductase Inhibition 192 (AUG)

Sulindac in Established Experimental Diabetes: a Follow-up Study 198 (AUG)

Dissociative Disorder

"Hysteria" in Clinical Neurology 101 (MAY)

Dopamine Mechanisms

Paradoxical Autonomic Response to Procyclidine in the Neuroleptic Malignant Syndrome 244 (AUG)

Dystonia

Writer's Cramp and Tremor Due to Brain Tumor 59 (FEB)

Psychogenic Dystonia: A Review of 18 Cases 136 (MAY)

Editorial

Editorial 3 (FEB)

Electroencephalography

EEG Results are Rarely the Same if Repeated within Six Months in Childhood Epilepsy 297 (NOV)

Electromyography

Electrical Stimulation of the Human Descending Motor Tracts at Several Levels 36 (FEB)

Levodopa Reduces Muscle Tone and Lower Extremity Tremor in Parkinson's Disease 280 (NOV)

Electrophysiologic

Electrical Stimulation of the Human Descending Motor Tracts at Several Levels 36 (FEB)

Encephalitis

Sudden 'Stroke-Like' Onset of Hemiparesis Due to Herpetic Encephalitis 320 (NOV)

Environment

Environmental Exposures in Elderly Canadians with Parkinson's Disease 232 (AUG)

Long term Exposure to Manganese in Rural Well Water Has No Neurological Effects 286 (NOV)

Sudden Death in Multiple Sclerosis Associated with Sun Exposure: A Report of Two Cases 305 (NOV)

Epidemiology

Familial Parkinson's Disease A Clinical Genetic Analysis 272 (NOV)

Long term Exposure to Manganese in Rural Well Water Has No Neurological Effects 286 (NOV) 
Epilepsy

Pyridoxine Dependent Epilepsy with Iatrogenic Sensory Neuronopathy 50 (FEB)

Cholinergic Mechanisms in Generalized Seizures: Importance of the Zona Incerta 116 (MAY)

EEG Results are Rarely the Same if Repeated within Six Months in Childhood Epilepsy 297 (NOV)

Genetics

Huntington's Disease: Recent Advances in Diagnosis and Management 5 (FEB)

Loss of Heterozygosity Analysis of Chromosomes 9, 10 and 17 in Gliomas in Families 17 (FEB)

Risk of Intracranial Aneurysms in Families with Subarachnoid Hemorrhage 12! (MAY)

Familial Parkinson's Disease: A Clinical Genetic Analysis 272 (NOV)

Glioma

Loss of Heterozygosity Analysis of Chromosomes 9, 10 and 17 in Gliomas in Families 17 (FEB)

$\mathrm{O}^{6}$-Methylguanine-DNA Methyltransferase in Tumors and Cells of the Oligodendrocyte Lineage 111 (MAY)

\section{Head Trauma}

$\beta$-Endorphin in Multiple Trauma Victims 160 (MAY)

Delayed Myelopathy After Trivial Neck Injury in a Patient With a Cervical Neurenteric Cyst 168 (MAY)

Vertebral Artery Injury Associated with a Jefferson Fracture 308 (NOV)

Headache

The Prognosis and Treatment of Headaches in Children - a 10 Year Follow-Up 47 (FEB)

Hemorrhage

Surgery of Unruptured, Asymptomatic Aneurysms: A Decision Analysis 30 (FEB)

Ruptured and Unruptured Intracranial Aneurysms-Surgical Outcome 187 (AUG)

Herniation

Brain Herniation: A Revision of Classical Concepts 83 (MAY)

Herpes Simplex

Sudden 'Stroke-Like' Onset of Hemiparesis Due to Herpetic Encephalitis 320 (NOV)

HIV-1

HIV-1 Associated Dementia: Clinical Features and Pathogenesis 92 (MAY)

Huntington's Disease

Huntington's Disease: Recent Advances in Diagnosis and Management 5 (FEB)

\section{Hypoglycemia}

Bilateral Temporal Lobe MRI Changes in Uncomplicated Hypoglycemic Coma 56 (FEB)

Hysteria

"Hysteria" in Clinical Neurology 101 (MAY)
Infectious Diseases

"Unexplained" Delayed Death From Fungal Meningitis After Meningioma Resection 239 (AUG)

Sudden 'Stroke-Like' Onset of Hemiparesis Due to Herpetic Encephalitis 320 (NOV)

Interferon

Indications for Interferon Beta IB Treatment in Multiple Sclerosis 334 (NOV)

Ischemic Brain Injury

Experimental Delayed Postischemic Spinal Cord Hypoperfusion After Aortic Crossclamping 202 (AUG)

Jones, Ernest

A History of Neurology in Toronto 1892 1960: Part I 322 (NOV)

Journal Name

Letter to the Editor: What's in a Name? 247 (AUG)

Reply from the Editor 247 (AUG)

Levodopa

Levodopa Reduces Muscle Tone and Lower Extremity Tremor in Parkinson's Disease 280 (NOV)

Magnetic Resonance Imaging

Bilateral Temporal Lobe MRI Changes in Uncomplicated Hypoglycemic Coma 56 (FEB)

Magnetic Stimulation

The Excitability of Human Corticospinal Neurons is Depressed by Thiopental 218 (AUG)

Manganese

Long term Exposure to Manganese in Rural Well Water Has No Neurological Effects 286 (NOV)

Memory

Neurobiology of Working Memory 75 (FEB) Reply 76 (FEB)

Meningiomas

"Unexplained" Delayed Death From Fungal Meningitis After Meningioma Resection 239 (AUG)

Metastatic Tumours

Brachytherapy for Recurrent Single Brain Metastasis 13 (FEB)

Myers, Donald Campbel

A History of Neurology in Toronto 1892 . 1960: Part I 322 (NOV)

Migraine

The Prognosis and Treatment of Headaches in Children - a 10 Year Follow-Up 47 (FEB)

Motor Control

Preparatory Postural Adjustments in Parkinsonian Patients with Postural Instability 126 (MAY)

Myoclonic-like Finger Microdisplacements in Patients with Cerebellar Deficits 144 (MAY)
The Excitability of Human Corticospinal Neurons is Depressed by Thiopental 218 (AUG)

Motor Evoked Potential

Electrical Stimulation of the Human Descending Motor Tracts at Several Levels 36 (FEB)

Movement Disorders

Huntington's Disease: Recent Advances in Diagnosis and Management 5 (FEB)

Writer's Cramp and Tremor Due to Brain Tumor 59 (FEB)

Preparatory Postural Adjustments in Parkinsonian Patients with Postural Instability 126 (MAY)

Psychogenic Dystonia: A Review of 18 Cases I36 (MAY)

Familial Parkinson's Disease: A Clinical Genetic Analysis 272 (NOV)

Levodopa Reduces Muscle Tone and Lower Extremity Tremor in Parkinson's Disease 280 (NOV)

Multiple Sclerosis

Fatigue in Multiple Sclerosis 75 (FEB)

Long Term Intrathecal Baclofen Therapy in Patients with Intractable Spasticity 208 (AUG)

Screening for Major Depression in the Early Stages of Multiple Sclerosis 228 (AUG)

Sudden Death in Multiple Sclerosis Associated with Sun Exposure: A Report of Two Cases 305 (NOV)

Indications for Interferon Beta IB Treatment in Multiple Sclerosis 334 (NOV)

Myelopathy

Delayed Myelopathy After Trivial Neck Injury in a Patient With a Cervical Neurenteric Cyst 168 (MAY)

Neonatal

Sterotactic Insertion of an Ommaya Reservoir: Technical Note 235 (AUG)

Neurenteric Cyst

Delayed Myelopathy After Trivial Neck Injury in a Patient With a Cervical Neurenteric Cyst 168 (MAY)

Neuro-Oncology

Brachytherapy for Recurrent Single Brain Metastasis 13 (FEB)

Loss of Heterozygosity Analysis of Chromosomes 9, 10 and 17 in Gliomas in Families 17 (FEB)

Writer's Cramp and Tremor Due to Brain Tumor 59 (FEB)

$\mathrm{O}^{6}$-Methylguanine-DNA Methyltransferase in Tumors and Cells of the Oligodendrocyte Lineage 111 (MAY)

Sterotactic Insertion of an Ommaya Reservoir: Technical Note 235 (AUG)

Neuro-Ophthalmology

Bilateral Central Ptosis in Acquired Immunodeficiency Syndrome 52 (FEB) 
Neuroimmunology

Indications for Interferon Beta IB Treatment in Multiple Sclerosis 334 (NOV)

Neuroleptic Maliganant Syndrome

Paradoxical Autonomic Response to Procyclidine in the Neuroleptic Malignant Syndrome 244 (AUG)

Neurology - Paediatric

The Prognosis and Treatment of Headaches in Children - a 10 Year Follow-Up 47 (FEB)

Neuropathology

Selective Sparing of Human Nucleus Accumbens in Aging and Anoxia 290 (NOV)

Experimental Study of NADPH-Diaphorase Positive Neurons in Nucleus Accumbens of Rats 294 (NOV)

Sudden Death in Multiple Sclerosis Associated with Sun Exposure: A Report of Two Cases 305 (NOV)

Neuropeptides

$\beta$-Endorphin in Multiple Trauma Victims 160 (MAY)

Neuropharmacology

Intrathecal Baclofen Therapy for Adults with Spinal Spasticity: Therapeutic Efficacy and Effect on Hospital Admissions 22 (FEB)

Cholinergic Mechanisms in Generalized Seizures: Importance of the Zona Incerta 116 (MAY)

Long Term Treatment of Intractable Reflex Sympathetic Dystrophy with Intrathecal Morphine 153 (MAY)

Nerve Microvessel Changes in Diabetes are Prevented by Aldose Reductase Inhibition 192 (AUG)

Sulindac in Established Experimental Diabetes: a Follow-up Study 198 (AUG)

Long Term Intrathecal Baclofen Therapy in Patients with Intractable Spasticity 208 (AUG)

The Excitability of Human Corticospinal Neurons is Depressed by Thiopental 218 (AUG)

An Open Trial of Pyridostigmine in Postpoliomyelitis Syndrome 223 (AUG)

Paradoxical Autonomic Response to Procyclidine in the Neuroleptic Malignant Syndrome 244 (AUG)

Levodopa Reduces Muscle Tone and Lower Extremity Tremor in Parkinson's Disease 280 (NOV)

Neurophysiology

Electrical Stimulation of the Human Descending Motor Tracts at Several Levels 36 (FEB)

Neuroprotection Therapy

Protection of the Brain after Aneurysmal Rupture 177 (AUG)

Neurosurgery

Surgery of Unruptured, Asymptomatic Aneurysms: A Decision Analysis 30 (FEB)
Sterotactic Insertion of an Ommaya Reservoir: Technical Note 235 (AUG)

\section{Nucleus Accumbens}

Selective Sparing of Human Nucleus Accumbens in Aging and Anoxia 290 (NOV)

Experimental Study of NADPH-Diaphorase Positive Neurons in Nucleus Accumbens of Rats 294 (NOV)

Ocular Motor

Bilateral Central Ptosis in Acquired Immunodeficiency Syndrome 52 (FEB)

Ommaya Reservoir

Sterotactic Insertion of an Ommaya Reservoir: Technical Note 235 (AUG)

Pain

Long Term Treatment of Intractable Reflex Sympathetic Dystrophy with Intrathecal Morphine 153 (MAY)

Parkinson's Disease

Preparatory Postural Adjustments in Parkinsonian Patients with Postural Instability 126 (MAY)

Environmental Exposures in Elderly Canadians with Parkinson's Disease 232 (AUG)

Familial Parkinson's Disease: A Clinical Genetic Analysis 272 (NOV)

Levodopa Reduces Muscle Tone and Lower Extremity Tremor in Parkinson's Disease 280 (NOV)

Long term Exposure to Manganese in Rural Well Water Has No Neurological Effects 286 (NOV)

Peripheral Neuropathy

Nerve Microvessel Changes in Diabetes are Prevented by Aldose Reductase Inhibition 192 (AUG)

Sulindac in Established Experimental Diabetes: a Follow-up Study 198 (AUG)

Peripheral Neuropathy

Sensorimotor Neuropathy and Abnormal Vitamin $B_{12}$ Metabolism in Early HIV Infection 43 (FEB)

Pyridoxine Dependent Epilepsy with latrogenic Sensory Neuronopathy 50 (FEB)

Nerve Microvessel Changes in Diabetes are Prevented by Aldose Reductase Inhibition 192 (AUG)

Sulindac in Established Experimental Diabetes: a Follow-up Study 198 (AUG)

Isolated Suprascapular Nerve Palsy: a Review of Nine Cases 301 (NOV)

Poliomyelitis

An Open Trial of Pyridostigmine in Postpoliomyelitis Syndrome 223 (AUG)

Psychiatry

"Hysteria" in Clinical Neurology 101 (MAY)

Psychogenic Dystonia: A Review of 18 Cases 136 (MAY)

Screening for Major Depression in the Early Stages of Multiple Sclerosis 228 (AUG)
Ptosis

Bilateral Central Ptosis in Acquired Immunodeficiency Syndrome 52 (FEB)

\section{Pyridostigmine}

An Open Trial of Pyridostigmine in Postpoliomyelitis Syndrome 223 (AUG)

Radiation Oncology

Brachytherapy for Recurrent Single Brain Metastasis 13 (FEB)

Radiosurgery

Brachytherapy for Recurrent Single Brain Metastasis 13 (FEB)

Recovery

Mechanisms Underlying Functional Recovery Following Stroke 257 (NOV)

Rehabilitarion

Mechanisms Underlying Functional Recovery Following Stroke 257 (NOV)

Somatoform Disorder

"Hysteria" in Clinical Neurology 101 (MAY)

Psychogenic Dystonia: A Review of 18 Cases 136 (MAY)

Spasticity

Intrathecal Baclofen Therapy for Adults with Spinal Spasticity: Therapeutic Efficacy and Effect on Hospital Admissions 22 (FEB)

Long Term Intrathecal Baclofen Therapy in Patients with Intractable Spasticity 208 (AUG)

\section{Spinal Cord}

Intrathecal Baclofen Therapy for Adults with Spinal Spasticity: Therapeutic Efficacy and Effect on Hospital Admissions 22 (FEB)

Dépôts Amylö̈des Intraligamentaires dans la Sténose du Canal Rachidien 164 (MAY)

Delayed Myelopathy After Trivial Neck Injury in a Patient With a Cervical Neurenteric Cyst 168 (MAY)

Experimental Delayed Postischemic Spinal Cord Hypoperfusion After Aortic Crossclamping 202 (AUG)

Long Term Intrathecal Baclofen Therapy in Patients with Intractable Spasticity 208 (AUG)

Spinal Cord Injury

Experimental Delayed Postischemic Spinal Cord Hypoperfusion After Aortic Crossclamping 202 (AUG)

Long Term Intrathecal Baclofen Therapy in Patients with Intractable Spasticity 208 (AUG)

Spinal Cord Physiology

The Excitability of Human Corticospinal Neurons is Depressed by Thiopental 218 (AUG)

Spinal Stenosis

Dépôts Amyloïdes Intraligamentaires dans la Sténose du Canal Rachidien 164 (MAY)

St. Michael's Hospital, Toronto

A History of Neurology in Toronto 1892 1960: Part I 322 (NOV) 
Stroke

Mechanisms Underlying Functional Recovery Following Stroke 257 (NOV)

Subarachnoid Hemorrhage

Risk of Intracranial Aneurysms in Families with Subarachnoid Hemorrhage 121 (MAY)

Protection of the Brain after Aneurysmal Rupture 177 (AUG)

Ruptured and Unruptured Intracranial Aneurysms-Surgical Outcome 187 (AUG)

Letter to Editor: Risk of Intracranial Aneurysms in Families with Subarachnoid Hemorrhage 333 (NOV)

Reply 333 (NOV)

Suprascapular Nerve

lsolated Suprascapular Nerve Palsy: a Review of Nine Cases 30l (NOV)

Sympathetic Dystrophy

Long Term Treatment of Intractable Reflex Sympathetic Dystrophy with Intrathecal Morphine 153 (MAY)
Therapeutics

Canadian Guidelines for the Development of Antidementia Therapies: a Conceptual Summary 62 (FEB)

Toronto General Hospital

A History of Neurology in Toronto 1892 1960: Part I 322 (NOV)

Toxicology

Environmental Exposures in Elderly Canadians with Parkinson's Disease 232 (AUG)

Long term Exposure to Manganese in Rural Well Water Has No Neurological Effects 286 (NOV)

Trauma

Isolated Suprascapular Nerve Palsy: a Review of Nine Cases 301 (NOV)

Vertebral Artery Injury Associated with a Jefferson Fracture 308 (NOV)

Tremor

Writer`s Cramp and Tremor Due to Brain Tumor 59 (FEB)

\section{Vertebral A rtery}

Vertebral Artery Injury Associated with a Jefferson Fracture 308 (NOV)

Virology

Sudden 'Stroke-Like' Onset of Hemiparesi Due to Herpetic Encephalitis 320 (NOV)

\section{Virus Infections}

Sensorimotor Neuropathy and Abnormal Vitamin $B_{12}$ Metabolism in Early HIV Infection 43 (FEB)

HIV-1 Associated Dementia: Clinical Features and Puthogenesis 92 (MAY)

Vitamin

Sensorimotor Neuropathy and Abnormal Vitamin $B_{12}$ Metabolism in Early HIV Infection 43 (FEB)

Pyridoxine Dependent Epilepsy with latrogenic Sensory Neuronopathy 50 (FEB)

\section{THE CANADIAN JOURNAL OF NEUROLOGICAL SCIENCES}

\section{VOL. 22, NO. 1 FEB. 1995}

Message from the Edito

Editorial - New Canadian Initiatives in Dementia Research

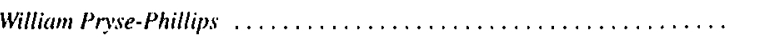

\section{REVIEW ARTICLE}

Huntington's Disease: Recent Advances in Diagnosis and Management

Sarah Furtado and Okasana Suchowersky

ORIGINAL ARTICLES

Brachytherapy for Recurrent Single Brain Metastasis

Mark Bernstein. Alberto Cabantog. Normand Laperricre,

Phil Leang and Cindy Thomason . . . . . . . . . . . . . . . . . . . . .

Loss of Hetcrozygosity Analysis of Chromosomes 9, 10 and 17 in

Glimas in Families

Christopher J Watling. Donald J van Meyel, David A Ransay,

David R Macdonald and J Gregory Cairncross ..................

Intrathecal Baclofen Therapy for Adults with Spinal Spasticity:

Therapeutic Eflicacy and Effect on Hospital Admissions

Patricia Nance, Orpha Schryvers. Brian Schmidt. Hy Dubo.

Brenda Loveridge and Derek Fewer . ..................

Surgery of Unruptured, Asymptomatic Aneurysms: a Decision Analysis Richard Leblanc and Keith J Worsley .....................

Electrical Stimultation of the Human Descending Motor Tracts at Several Levels Yoshikazu Ugawa, Kickko Genba-Shimizu and Ichiro Kanazawa .......

Sensorimotor Neuropathy and Abnormal Vitamin $B_{12}$ Metabolism in

Early HIV Infection $M$ Veilleux, $O$ Pallicl and I Faluz

Joseph Dooley and Alexa Bagnell . . . . . . . . . . . . . . . .

Pyridoxine Dependent Epilepsy with Iatrogenic Sensory Neuronopathy Richard S Mclachlan and William F Brown ................

Bilateral Central Ptosis in Acquired Immunodeficiency Syndrome

Jason JS Barton, Randy H Kardon, Daniel Slagel and

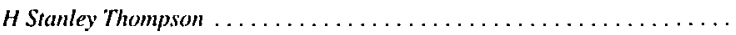

Bilateral Temporal Lobe MRI Chagnes in Uncomplicated Hypoglycemic Coma BF Boeve, DG Bell and JH Noseworthy . . . . . . . . . . . . . . . . .

Writer's Cramp and Tremor Due to Brain Tumor I Mllanov and D Georgiev

\section{NEUROLOGICAL PRACTICE}

Canadian Guidelines for the Development of Antidementia Therapies;

a Conceptual Summary

Erich Mohr, Howard Feldman and Serge Gauthier ........... 62

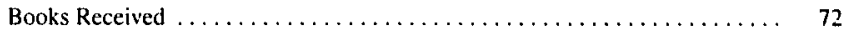

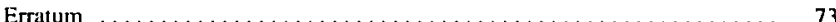

CORRESPONDENCE

Fatigue in Multiple Sclerosis Ernest Friedman

Reply

CJ Archibald and JD Fisk .......................... 75

Nemobiology of Working Menory

Ernest Friedman $\ldots \ldots \ldots \ldots \ldots \ldots \ldots \ldots \ldots \ldots \ldots \ldots \ldots \ldots, \ldots \ldots$

Reply

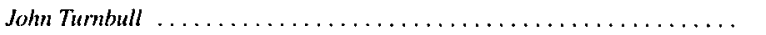

VOL. 22, NO. 2 MAY 1995

REVIEW AR'TICLES

Brain Herniation: A Revision of Classical Concepts

C Miller Fisher

HIV-1 Associated Dementia: Clinical Features and Pathogenesis Chirstopher Power and Richand T Johnson ...............

"Hysteria" in Clinical Neurology

François MMai ................................... 10

ORIGINAL ARTICLES

$\mathrm{O}^{6}$-Methylguanine-DNA Methyltransferase in Tumors and Cells of the

Oligodendrocyte Lineage

Catherine L Nutt. Joseph F Costello, Linda L Bambrick, Daniel $B$ Yarosh Lode J Swimen, Ann F Chambers and J Gregon Cairncross . . . . . . . 111

Cholinergic Mechanisms in Generalized Seizures: Importance of the Zona Incerta Stefan M Brudzynski, James W Cruickshank and

Richard S McLachlan ............................. 116

Risk of Intracranial Aneurysms in Families with Subarachnoid Hemorrhage Mark J Alberts, Ana Quinones, Carmelo Graffagnino. Allan Friedman and Allen $D$ Roses ....................... 121

Preparatory Postural Adjustments in Parkinsonian Patients with Postural Instability Robert G Lee, Ida Tonolli, Francois Viallet, Roslyn Aurenty and Jean Massion 